\title{
Expresión de TGFß1 por Fibroblastos de Mucosa Oral Expuestos a Radiación Ultravioleta B
}

\author{
TGF1 Expression by Oral Mucosa Fibroblasts Exposed to Ultraviolet $\beta 1$ Radiation
}

\author{
Cubillos A. Paulina ${ }^{*+*}$; Claire Labrousse ${ }^{n+*}$; Paulina Zapata"
}

CUBILLOS, A. P.; LABROUSSE, C.; ZAPATA, P.; VENEGAS, B. \& ROJAS, I. G. Expresión de TGF $\beta 1$ por fibroblastos de mucosa oral expuestos a radiación ultravioleta B. Int. J. Odontostomat., 10(3):449-454, 2016.

RESUMEN: El objetivo de este estudio fue evaluar el efecto de la radiación ultravioleta (UV) B sobre la expresión del factor de crecimiento transformante (TGF) $\beta 1$ por fibroblastos de mucosa oral, con el objetivo de dilucidar si este tipo celular puede contribuir a la expresión de TGF $\beta 1$ en bermellón labial sobreexpuesto a la radiación UV. Se obtuvieron cultivos primarios de fibroblastos desde explantes de mucosa bucal, los que fueron irradiados con una dosis única de luz UVB (60 $\mathrm{mJ} / \mathrm{cm}^{2}$ ). Se midió proliferación celular con el método MTT, y la expresión de TGF $\beta 1$, a nivel de ARN mensajero (normalizado a GAPDH) por RT-PCR y a nivel de proteína mediante inmunofluorescencia. Se observó una disminución de la proliferación celular de los fibroblastos de mucosa oral a las 24 hrs post-irradiación en relación a los fibroblastos no irradiados $(P<0,05$, Mann Whitney). No se encontraron diferencias entre los fibroblastos control y los irradiados en la expresión de TGF $\beta-1$ ni a nivel de mensajero (0,5 y 6 h post-irradiación), ni de proteína (24 h post-irradiación). Los resultados sugieren que los fibroblastos de mucosa oral presentan una disminución de su proliferación en respuesta a una dosis única de radiación UVB, sin que se afecte la expresión de TGF $\beta-1$, la que fue similar a los fibroblastos no irradiados. Esto sugiere que los fibroblastos contribuirían a la producción de TGF $\beta$-1 en respuesta a la exposición crónica a UVB del bermellón labial.

PALABRAS CLAVE: fibroblastos, luz UVB, TGF-beta, proliferación.

\section{INTRODUCCIÓN}

El bermellón labial es un tejido de transición entre piel y mucosa intraoral, donde tanto las células del epitelio como de la lámina propia (ej. queratinocitos, fibroblastos, mastocitos, células endoteliales, etc.) están expuestas a estímulos medioambientales incluyendo la luz ultravioleta (UV) solar (Lundeen et al., 1985; Winning et al., 2000). La radiación UVB (290$320 \mathrm{~nm}$ ) constituye menos de un $5 \%$ de la radiación ultravioleta que alcanza la superficie terrestre, sin embargo es la principal responsable de los efectos nocivos de la luz solar, que incluyen fotoenvejecimiento y cáncer (Bowden et al., 2004). Una de las características del fotoenvejecimiento de piel y labios es la elastosis solar, que se debe principalmente al depósito anormal de elastina y en menor medida también a la degradación del colágeno y elastina pre-existentes
(Bhawan et al., 1995). Estudios previos sugieren que la principal célula asociada con la elastosis solar en el labio es el fibroblasto, sin embargo, no se conocen los mecanismos involucrados (Rojas et al., 2012).

El factor de crecimiento transformante beta (TGF) $\beta 1$ es reconocido como el principal factor regulador de la síntesis de colágeno y elastina en fibroblastos de piel y se cree que es el responsable del aumento de elastina en pieles fotodañadas (Kähäri et al., 1992; Kossodo et al., 2004). En el labio, el contenido de TGF $\beta 1$ está aumentado en queilitis actínica comparado con mucosa sana, sin embargo, los tipos celulares que lo producen no han sido descritos (Gasparoto et al., 2014). Estudios previos han mostrado que la exposición a la radiación UVB, puede tanto

\footnotetext{
Depto. de Patología y Diagnóstico, Facultad de Odontología, Universidad de Concepción, Concepción, Chile.

" Magister en Ciencias Biomédicas, Mención Patología Oral, Universidad de Talca, Talca, Chile.

*** Departamento de Estomatología Quirúrgica y Facultad de Odontología, Universidad de Concepción, Concepción, Chile.

**** Escuela de Odontología, Universidad de Talca, Talca, Chile.

***** Department of Periodontics and Endodontics, University at Buffalo School of Dental Medicine EE. UU.

Estudio financiado por proyecto FONDECYT 1090287 (IP Dr. IG Rojas), CONICYT Chile.
} 
disminuir como aumentar la expresión de TGF $\beta 1$ en fibroblastos de dermis humana (Debacq-Chainiaux et al., 2005; Hwang et al., 2013). En fibroblastos de mucosa oral, la exposición a productos bacterianos, como el lipopolisacárido (LPS), estimula la expresión de TGF $\beta 1$, sin embargo, el efecto de estímulos medioambientales, como la radiación UVB, sobre su producción no se conoce (Azevedo et al., 2014).

El objetivo de este estudio fue analizar el efecto de la exposición a una dosis subcitotóxica de radiación ultravioleta B sobre la expresión de TGF $\beta 1$ por cultivos primarios de fibroblastos establecidos desde explantes de mucosa oral humana.

\section{MATERIAL Y MÉTODO}

Cultivos primarios de fibroblastos de mucosa oral. Se obtuvieron cultivos primarios de fibroblastos a partir de biopsias de mucosa oral cercana a la comisura labial de dos pacientes jóvenes (sexo masculino y femenino, menores de 25 años), no fumadores y sin enfermedades sistémicas a través del método del explante ya descrito (Smith et al., 2004). Se obtuvo el consentimiento informado de los pacientes. El estudio fue aprobado por el Comité de Bioética de la Universidad de Concepción. Las células fueron caracterizadas por inmunofluorescencia. Los experimentos se realizaron usando las células en cultivo entre el cuarto y octavo pasaje.

Inmunofluorescencia. Los fibroblastos de mucosa oral (FMO) se sembraron sobre cubreobjetos en placas de 24 pocillos a una concentración de $5 \times 104$ células/pocillo, en medio DMEM completo y se incubaron $24 \mathrm{~h}$ a $37^{\circ} \mathrm{C}$ y $5 \% \mathrm{CO}_{2}$. Se utilizaron los anticuerpos (Ac) primarios monoclonales anti-prolil-4-hidroxilasa (1:50, Chemicon, Millipore, Temecula, CA), anticitokeratina (CK) (1:50, Dako, Carpintería, CA) y policlonal anti-TGF- $\beta$ (Thermo, Rockford, IL). Se utilizaron los Ac secundarios Goat anti-mouse IgG acoplado a FITC (KPL, Gaithersburg, MD) y Swine anti-rabbit acoplado a TRITC (Dako). Se utilizó medio de montaje con DAPI para tinción nuclear (Vector, Burligame, CA). En cada experimento se descartó la contaminación por micoplasma utilizando tinción de ADN con DAPI (Uphoff et al., 1992). Las muestras se analizaron con un microscopio de epifluorescencia, Nikon Eclipse 50i conectado a una cámara digital Nikon (DS-5M) y se utilizó el programa de captura de imágenes Nis-Element F2.20 (Nikon Instruments, Inc., Melville, NY).
Irradiación de cultivos primarios con luz ultravioleta (UV)B. Para la irradiación con luz UVB se utilizó una lámpara UVB Philips FS40 protegida con un filtro UVC Kodacell y la dosis de $60 \mathrm{~mJ} / \mathrm{cm}^{2}$ se corroboró con un radiómetro UVX digital de acuerdo al protocolo ya descrito (Boza et al., 2010). Los fibroblastos controles fueron sometidos a los mismos cambios de medio de los fibroblastos irradiados, pero no fueron expuestos a la radiación UVB (Boza et al.).

Ensayo de proliferación MTT. Se utilizó el ensayo colorimétrico MTT (3-(4, 5-dimethylthiazolyl-2)-2, 5diphenyltetrazolium bromide) (Vybrant MTT Cell Proliferation assay Kit; Molecular Probes, Invitrogen). Los fibroblastos fueron cultivados en placas de 96 pocillos a una densidad de $1 \times 10^{6}$ células $/ \mathrm{ml}$ y fueron irradiadas con luz UVB $\left(60 \mathrm{~mJ} / \mathrm{cm}^{2}\right)$. La proliferación se midió a las $24 \mathrm{~h}$ post-irradiación. La lectura colorimétrica se realizó a $540 \mathrm{~nm}$ en un lector de ELISA (Multiskan EX, Thermo Corporation, USA). Los resultados fueron expresados en unidades densitométricas.

Aislamiento de RNA total y RT-PCR. El aislamiento de ARN y RT-PCR se realizaron de acuerdo a los métodos previamente descritos (Boza et al.). Los fibroblastos fueron cultivados a una densidad de $6 \times 10^{5}$ células $/ \mathrm{ml}$ en placas de $10 \mathrm{~mm}$ en $10 \mathrm{~mL}$ de medio DMEM completo. El RNA se aisló con Trizol (Invitrogen, Rockville, MD), y se realizó la transcripción reversa del RNA total y PCR utilizando partidores para TGF- $\beta 1$ forward 5 '-CCC ACA ACG AAA TCT ATG ACAA-3', reverse 5'-AAG ATAACC ACT CTG GCG AGT C-3'(246 bp) (Ma et al., 2013); y GAPDH forward 5'-CGG AGT CAA CGG ATT TGG TCG TAT-3', reverse 5'-AGC CTT CTC CAT GGT TGG TGA AGA C-3' (310 bp) (Baram et al., 2001). Los productos del PCR fueron separados electroforéticamente en un gel de agarosa al 1,5\% teñido con bromuro de etidio $0,1 \mu \mathrm{g} / \mathrm{ml}$ y se visualizaron con un transiluminador acoplado a un sistema de imágenes (DP-001, Viber, Lourmat, Francia). Las bandas fueron analizadas densitométricamente en el programa IMAGE J $(\mathrm{NIH}$, Bethesda, MD) y se obtuvo la expresión de TGF $\beta 1$ normalizada a GAPDH para controles e irradiados en cada experimento. Se utilizó el promedio de expresión de los fibroblastos controles como el $100 \%$ y se obtuvo el porcentaje de expresión de TGF $\beta 1$ de los fibroblastos irradiados en relación al promedio del control.

Análisis estadístico. Se utilizó el programa JMP-IN 4.0.4 (SAS Institute, USA). Las diferencias entre los grupos se analizaron usando Mann-Whitney o $t$-test según la distribución de los datos. Se consideró como significativo un valor $p<0,05$. 
CUBILLOS, A. P.; LABROUSSE, C.; ZAPATA, P.; VENEGAS, B. \& ROJAS, I. G. Expresión de TGFß1 por fibroblastos de mucosa oral expuestos a radiación ultravioleta B. Int. J. Odontostomat., 10(3):449-454, 2016.

\section{RESULTADOS}

Efecto de la irradiación UVB sobre la expresión de marcadores de fibroblastos y proliferación de cultivos primarios de fibroblastos de mucosa oral. Los cultivos primarios de FMO mostraron expresión de vimentina y prolil-4 hidroxilasa tanto en presencia o ausencia de una dosis única de luz UVB $\left(60 \mathrm{~mJ} / \mathrm{cm}^{2}\right)$ (Fig. 1). Se comprobó además que no había contaminación de los cultivos con queratinocitos (Fig. 1). Se evaluó si el efecto de la exposición a una dosis única de luz UVB disminuía la proliferación de FMO, encontrándose una disminución de la proliferación a las 24 h post-irradiación ( $P<0.05$, Mann-Whitney) (Fig. 2), de acuerdo a lo descrito previamente (Boza et al.).
Detección de la expresión de TGF $\beta 1$ por fibroblastos de mucosa oral. A nivel de ARN mensajero, se observó una expresión similar de TGF $\beta 1$ en fibroblastos control e irradiados a las $6 \mathrm{~h}$ post-irradiación (Fig. 3). Al observar expresión a nivel de proteína por inmunofluorescencia, a las $24 \mathrm{~h}$ post-irradiación ambos, fibroblastos irradiados y controles, fueron positivos para TGF $\beta 1$ (Fig. 4). EI TGF $\beta 1$ se observó tanto en el citoplasma como en el núcleo de los fibroblastos analizados.
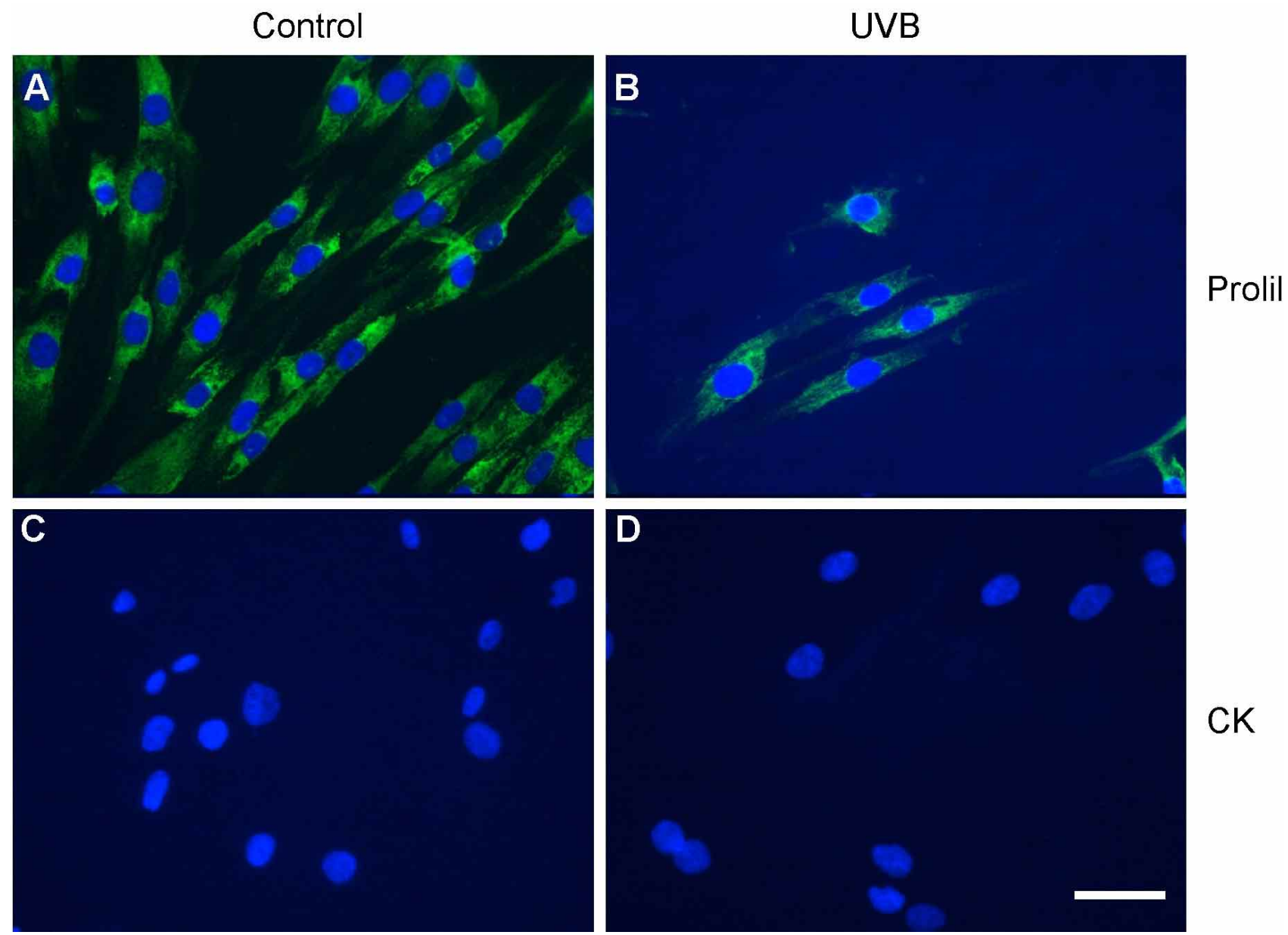

Fig. 1. Efecto de la radiación UVB sobre la expresión de marcadores de fibroblastos por parte de cultivos primarios de fibroblastos de mucosa oral (FMO). Fotomicrografías representativas, de 3 experimentos independientes, de FMO (5x10 céls/pocillo) procesados para la detección por inmunofluorescencia de prolil-4-hidroxilasa (a,b) y citoqueratina (c,d), a las 24 post-irradiación UVB $\left(60 \mathrm{~mJ} / \mathrm{cm}^{2}\right)(b, d)$ o placebo $(a, c)$. Se utilizaron anticuerpos secundarios acoplados a FITC, y tinción nuclear con DAPI. La barra representa $50 \mu \mathrm{m}$. 


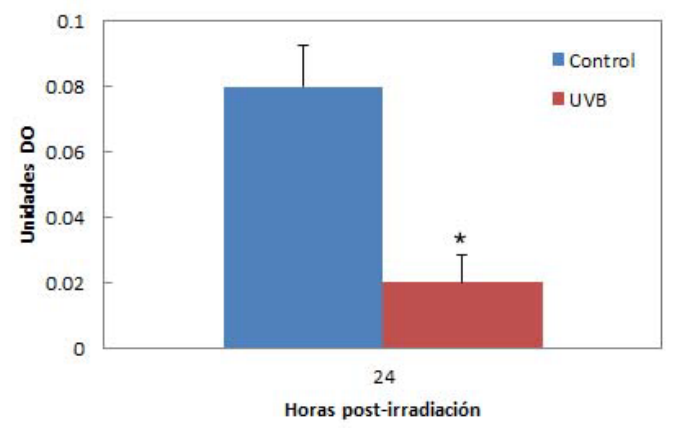

Fig. 2. Efecto de la radiación UVB sobre la proliferación de FMO. Fibroblastos $\left(1 \times 10^{5}\right.$ céls $\left./ \mathrm{ml}\right)$ se expusieron a una única dosis de radiación UVB $\left(60 \mathrm{~mJ} / \mathrm{cm}^{2}\right)$ o placebo, y su proliferación se evaluó con el ensayo MTT a las $24 \mathrm{hrs}$ post-irradiación. Los resultados se expresaron como el promedio de unidades de densidad óptica (DO) \pm DS (540nm) de dos experimentos combinados ( $n=6$ pocillos/grupo), que muestran resultados similares de a lo menos 5 experimentos previos. ${ }^{*} \mathrm{P}<0.05$ (Mann-Whitney) para fibroblastos irradiados comparados con los controles.

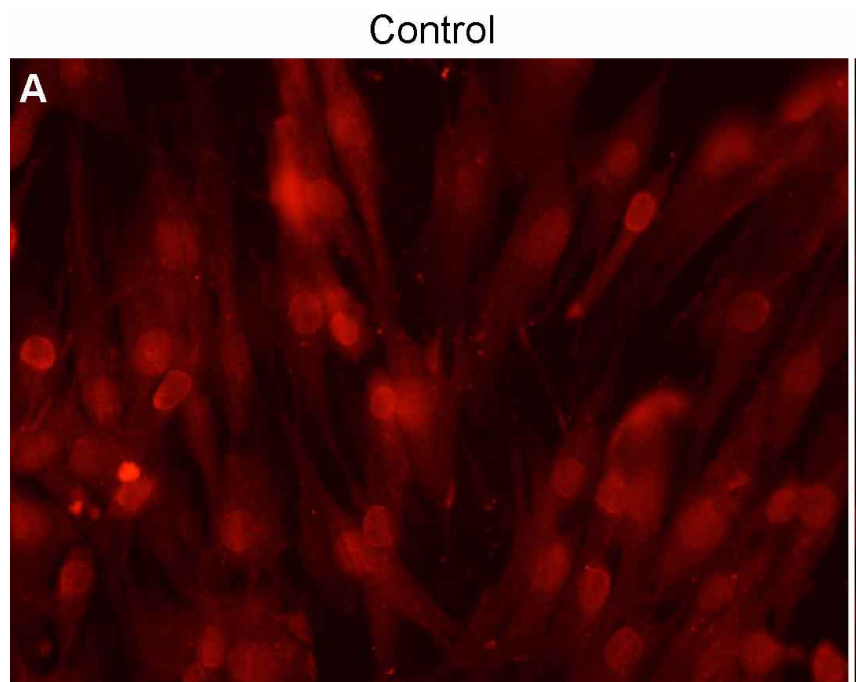

Fig. 4. Efecto de radiación UVB sobre la expresión de TGF- $\beta 1$ a nivel de proteína por FMO. Microfotografías representativas de fibroblastos ( $5 \times 10^{4}$ céls/pocillo) procesados para la detección de TGF $\beta 1$ por inmunofluorescencia (anticuerpo secundario acoplado a FITC). La barra representa $50 \mu \mathrm{m}$.

\section{DISCUSIÓN}

Según nuestro conocimiento este es el primer estudio en mostrar que los fibroblastos de mucosa oral humana expresan TGF $\beta 1$ a nivel de RNA mensajero y proteína independiente de la exposición a

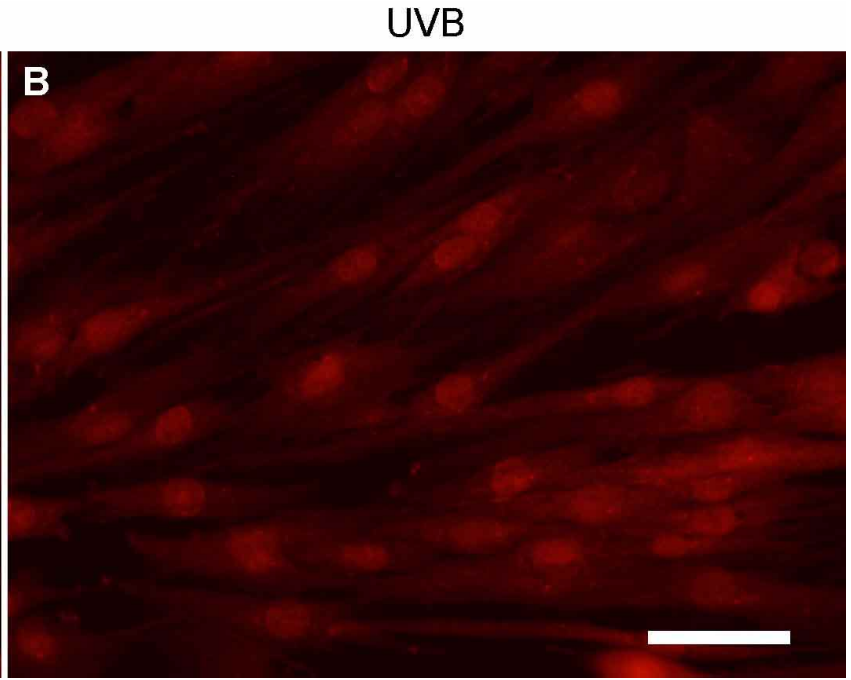

a)

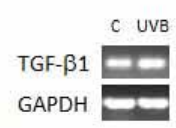

b)

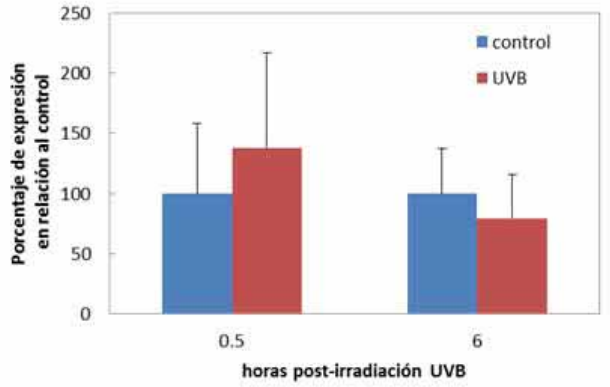

Fig. 3. Efecto de la radiación UVB sobre la expresión de RNA mensajero de TGF $\beta 1$ por FMO. Fibroblastos $\left(6 \times 10^{5}\right.$ céls/placa) fueron irradiados con $60 \mathrm{~mJ} / \mathrm{cm}^{2}$ de UVB o placebo. Se aisló el RNA total a las $6 \mathrm{~h}$ post-irradiación, y se analizó la expresión de TGFß1 (normalizado a GAPDH) por RT-PCR. (a) gel representativo a las 6 h post-irradición de 3 experimentos (b) Promedio \pm SEM del porcentaje de expresión de TGF $\beta 1$ por fibroblastos irradiados en relación al promedio de expresión de fibroblastos control normalizado a GAPDH ( $n=3-6$ experimentos).

B

una dosis única de radiación UVB. Además, de acuerdo a lo mostrado en estudios previos, los fibroblastos de mucosa oral tuvieron una reducción en su proliferación en respuesta a la exposición a una dosis sub- 
citotóxica de UVB, equivalente a 2-3 dosis mínimas de eritema (MED). Aunque el ensayo MTT mide viabilidad, también ha sido ocupado como un ensayo subrogante de proliferación, como se demostró previamente al obtener diferencias similares en proliferación de fibroblastos irradiados y controles utilizando el ensayo de incorporación de timidina tritiada (Boza et al.). Sin embargo, pese a la alteración en la proliferación, no hubo una disminución en la capacidad de expresar TGF $\beta 1$ por parte de FMO. No se encontraron diferencias entre fibroblastos control e irradiados en los niveles de expresión de mensajero para TGF $\beta 1$ a las 6 horas post-irradiación, y se observó expresión de TGF $\beta 1$ a nivel de proteína a las 24 horas post-irradiación.

Se ha descrito que existen diferencias entre fibroblastos de piel y mucosa oral durante la respuesta de cicatrización (Glim et al., 2013). Los fibroblastos de mucosa oral proliferan más rápido que los de piel al ser cultivados in vitro, y producen más colágeno al ser estimulados con TGF $\beta$ (Lee et al., 1999). Sin embargo, en respuesta a la irradiación con luz UVB, tanto los fibroblastos de mucosa oral como de piel muestran una reducción en su proliferación (DebacqChainiaux et al.; Boza et al.). Nuestros resultados son similares a los de Choi et al. (2006) que mostraron que fibroblastos de dermis humana irradiados con una dosis de luz UVB de banda angosta, equivalente a la de este estudio, expresaban niveles similares de mensajero y proteína para TGF $\beta 1 \mathrm{com}$ parados con fibroblastos control en las primeras 12 horas post-irradiación. Hwang et al. observaron una reducción de la expresión de TGF $\beta 1$ a nivel de proteína en fibroblastos de piel humana a las 24 horas post-irradiación, sin embargo, utilizaron una dosis de radiación UVB dos veces mayor que la de nuestro estudio (144 $\left.\mathrm{mJ} / \mathrm{cm}^{2}\right)$.

Los fibroblastos tienen un rol fundamental en la respuesta a la radiación UV. Son las células responsables de producir elastina, llevando a la formación de elastosis solar, mecanismo que está mediado por TGF $\beta 1$ en la piel (Kahari et al.; Kossodo et al.). Además, el TGF $\beta 1$ contribuye a disminuir la respuesta proliferativa de fibroblastos en respuesta a la radiación UVB (Debacq-Chainiaux et al.). Nuestros resultados sugieren que los fibroblastos de mucosa oral expresan TGF $\beta 1$ y que una dosis única de UVB no disminuye su capacidad de expresión de esta citoquina. Ya que se ha descrito que hay un aumento de TGF $\beta 1$ en tejidos de piel y labio expuestos en forma crónica a la radiación solar, los resultados su- gieren que además de los fibroblastos otros tipos celulares podrían estar contribuyendo a un aumento de la expresión de TGF $\beta$, incluyendo a queratinocitos y mastocitos. Además, la interacción entre mastocitos y fibroblastos, podría estimular una mayor expresión de TGF $\beta 1$ por parte de ambos tipos celulares, in vivo, ya que los mastocitos regulan diversas funciones de los fibroblastos (Sarchio et al., 2012).

Nuestro estudio se enfocó en analizar la expresión temprana de TGF $\beta 1$ por parte de fibroblastos de mucosa oral en respuesta a una dosis subcitotóxica de UVB. Futuros estudios deberán analizar el efecto de UVB sobre la expresión de TGF $\beta 1$ en etapas más tardías a la exposición a UVB, en dosis únicas o múltiples.

\section{AGRADECIMIENTOS}

Estudio financiado por proyecto FONDECYT 1090287 (IP Dr. IG Rojas), CONICYT Chile.

CUBillos, A. P.; LABROUSSE, C.; ZAPATA, P.; VENEGAS, B. \& ROJAS, I. G. TGF $\beta 1$ expression by oral mucosa fibroblasts exposed to ultraviolet B radiation. Int. J. Odontostomat., 10(3):449-454, 2016.

ABSTRACT. The objective of this study was to characterize the effect of Ultraviolet (UV) B irradiation on the expression of transforming growth factor (TGF) $\beta 1$ by oral mucosa fibroblasts, in order to assess if these cells contribute to the production of TGF $\beta-1$ in UV-irradiated lip vermillion. Primary cultures of fibroblasts were obtained from oral mucosa explants, and were irradiated with a single dose of UVB light $\left(60 \mathrm{~mJ} / \mathrm{cm}^{2}\right)$. The effects of UVB radiation on cell proliferation was evaluated by the MTT method. The effects of UVB on the expression of TGF- $\beta 1$ was analyzed by RTPCR (normalized to GAPDH) and by immunofluorescence. The results showed a decrease in the proliferation of UVBirradiated fibroblasts as compared to controls at $24 \mathrm{~h}$ postirradiation $(p<0.05)$. No variations in the expression of TGF $\beta 1$, both at the mRNA and protein level, were observed between control and UVB-irradiated fibroblasts during the first $24 \mathrm{~h}$ after irradiation. Oral mucosa fibroblasts have reduced proliferation in response to a single dose of UVB, but their expression of TGF $\beta 1$ was not affected. This suggests that oral mucosa fibroblasts may contribute to the production of TGF $\beta 1$ in the lip vermillion independent of UVB exposure.

KEY WORDS: fibroblasts, UVB light, TGF beta, proliferation. 


\section{REFERENCIAS BIBLIOGRÁFICAS}

Azevedo, F. P.; Morandini, A. C. F.; Sipert, C. R.; Dionísio, T. J.; Santos, C. F.; Damante, C. A.; de Rezende, M. L. R.; Passanezi Sant'Ana, A. C. \& Greghi, S. L. A. Palatal mucosa derived fibroblasts present an adaptive behavior regarding cytokine secretion when grafted onto the gingival margin. B. M. C. Oral Health, 14:21, 2014.

Baram, D.; Vaday, G. G.; Salamon, P.; Drucker, I.; Hershkoviz, R. \& Mekori, Y. A. Human mast cells release metalloproteinase- 9 on contact with activated $\mathrm{T}$ cells: juxtacrine regulation by TNF-alpha. J. Immunol., 167(7):4008-16, 2001

Bhawan, J.; Andersen, W.; Lee, J.; Labadie, R. \& Solares, G. Photoaging versus intrinsic aging: a morphologic assessment of facial skin. J. Cutan. Pathol., 22(2):154-9, 1995.

Bowden, G. T. Prevention of non-melanoma skin cancer by targeting ultraviolet-B-light signalling. Nat. Rev. Cancer, 4(1):23-35, 2004.

Boza, Y.; Yefi, R.; Rudolph, M. I.; Smith, P. C.; Oberyszyn, T. M.; Tober, K. L. \& Rojas, I. G. Single Exposure of human oral mucosa fibroblasts to ultraviolet $B$ radiation reduces proliferation and induces COX-2 expression and activation. Rev. Clin. Periodoncia Implantol. Rehabil. Oral, 3(3):1237, 2010.

Choi, C. P.; Kim, Y. I.; Lee, J. W. \& Lee, M. H. The effect of narrowband ultraviolet $B$ on the expression of matrix metalloproteinase-1, transforming growth factor-beta1 and type I collagen in human skin fibroblasts. Clin. Exp. Dermatol., 32(2):180-5, 2007.

Debacq-Chainiaux, F.; Borlon, C.; Pascal, T.; Royer, V.; Eliaers, F.; Ninane, N.; Carrard, G.; Friguet, B.; de Longueville, F.; Boffe, S.; Remacle, J. \& Toussaint, O. Repeated exposure of human skin fibroblasts to UVB at subcytotoxic level triggers premature senescence through the TGF-beta1 signaling pathway. J. Cell Sci., 118(Pt. 4):743-58, 2005.

Gasparoto, T. H.; de Souza Malaspina, T. S.; Damante, J. H.; de Mello Jr., E. F.; Ikoma, M. R. V.; Garlet, G. P.; Costa, M. R. S. N.; Cavassani, K. A.; da Silva, J. S. \& Campanelli, A. P. Regulatory T cells in the actinic cheilitis. J. Oral Pathol. Med., 43(10):754-60, 2014.

Glim, J. E.; van Egmond, M.; Niessen, F. B.; Everts, V. \& Beelen, R. H. Detrimental dermal wound healing: what can we learn from the oral mucosa? Wound Repair Regen., 21(5):64860, 2013.

Hwang, E.; Kim, S. H.; Lee, S.; Lee, C. H.; Do, S. G.; Kim, J. \& Kim, S. Y. A comparative study of baby immature and adult shoots of Aloe vera on UVB-induced skin photoaging in vitro. Phytother. Res., 27(12):1874-82, 2013.
Kähäri, V. M.; Olsen, D. R.; Rhudy, R. W.; Carrillo, P.; Chen, Y. Q. \& Uitto, J. Transforming growth factor-beta up-regulates elastin gene expression in human skin fibroblasts. Evidence for post-transcriptional modulation. Lab. Invest., 66(5):5808, 1992.

Kossodo, S.; Wong, W. R.; Simon, G. \& Kochevar, I. E. Effects of UVR and UVR-induced cytokines on production of extracellular matrix proteins and proteases by dermal fibroblasts cultured in collagen gels\%. Photochem. Photobiol., 79(1):86-93, 2004.

Lundeen, R. C.; Langlais, R. P. \& Terezhalmy, G. T. Sunscreen protection for lip mucosa: a review and update. J. Am. Dent. Assoc., 111(4):617-21, 1985.

Ma, G. F.; Miao, Q.; Zeng, X. G. et al. Transforming growth factor-beta 1 and beta 2 in gastric precancerous and cancer and roles in tumor-cell interactions with peripheral blood mononuclear cells in vitro. PlosS One, 8(1):e54249, 2013.

Rojas, I. G.; Boza, Y. V.; Spencer, M. L.; Flores, M. \& Martínez, A. Increased fibroblast density in actinic cheilitis: association with tryptase-positive mast cells, actinic elastosis and epithelial p53 and COX-2 expression. J. Oral Pathol. Med., 41(1):27-33, 2012.

Sarchio, S. N.; Kok, L. F.; O'Sullivan, C.; Halliday, G. M. \& Byrne, S. N. Dermal mast cells affect the development of sunlightinduced skin tumours. Exp. Dermatol., 21(4):241-8, 2012.

Smith, P. C.; Santibáñez, J. F.; Morales, J. P. \& Martínez, J. Epidermal growth factor stimulates urokinase-type plasminogen activator expression in human gingival fibroblasts. Possible modulation by genistein and curcumi. J. Periodont. Res., 39(6):380-387, 2004.

Uphoff, C. C.; Gignac, S. M. \& Drexler, H. G. Mycoplasma contamination in human leukemia cell lines. I. Comparison of various detection methods. J. Immunol. Methods, 149(1):43-53, 1992.

Winning, T. A. \& Townsend, G. C. Oral mucosal embryology and histology. Clin. Dermatol., 18(5):499-511, 2000

Dirección para correspondencia:

Gina Rojas, DDS, PhD

Depto. de Estomatología Quirúrgica, Sección Periodoncia

Facultad de Odontología

Universidad de Concepción

Roosevelt 1550

Concepción

CHILE

E-mail: isolde.gina@gmail.com

Recibido : 06-07-2016

Aceptado: $17-10-2016$ 